ARGONNE NATIONAL LABORATORY

9700 South Cass Avenue

ANL/MCS-TM- -141

Argonne, Illinois 60439-4801

DE91 005214

ANL/MCS-TM-141

\title{
FORMED: An X Window System Application for Managing First-Order Formulas*
}

\author{
by \\ Tamara L. Henry** and William W. McCune \\ Mathematics and Computer Science Division \\ Technical Memorandum No. 141
}

November 1990

\footnotetext{
"This work was supported by the Applied Mathematical Sciences subprogram of the Office of Energy Research, U.S. Department of Energy, under Contract W-31-109-Eng-38.

**Participant in the Summer 1990 Student Research Participation Program. Curzent address: Department of Mrthematics, University of Tennessee, Knoxville, TN.
} 


\section{Contents}

Abstract 1

1 Introduction $\quad 2$

2 Syntax 3

2.1 Terms and Atoms 3

2.2 Formulas 4

$3 \quad$ Starting FORMED ED 4

4 The Display and Selection of Subformulas 5

$\begin{array}{llr}5 & \text { Logic Transformations } & 6\end{array}$

6 Editing Transformations $\quad 8$

7 The Undo/Redo Mechanism 10

8 The Display and Select Algorithms 11

9 Extension to Terms 12

$\begin{array}{ll}\text { References } & 12\end{array}$ 


\title{
FORMED: An X Window System Application for Managing First-Order Formulas
}

\author{
Tamara L. Henry and William W. McCune
}

\begin{abstract}
FORMED is a window-based program for constructing, displaying, and managing first-order logic formulas. The main motivation for constructing FORM ED was the desire to have formulas displayed in a readable, two-dimensional format. Users of FORM ED can make two kinds of transformation on formulas: (1) logic transformations, such as negation normal form translation, which preserve the meaning of a formula, and (2) edit transformations, which can be used to make arbitrary changes, such as adding a hypothesis to a subformula. FormED was written by using the $\mathrm{X}$ Window System, Version 11, and code from the theorem prover OTTER.
\end{abstract}




\section{Introduction}

Most computer scientists, logicians, and mathematicians would probably agree that

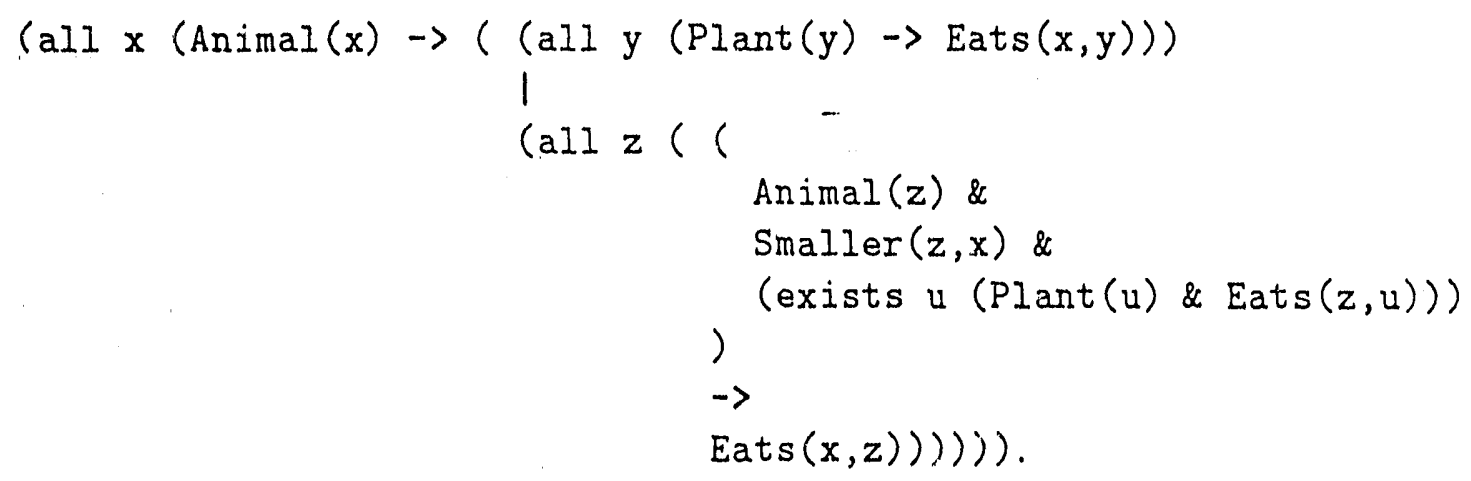

is more difficult to ponder than

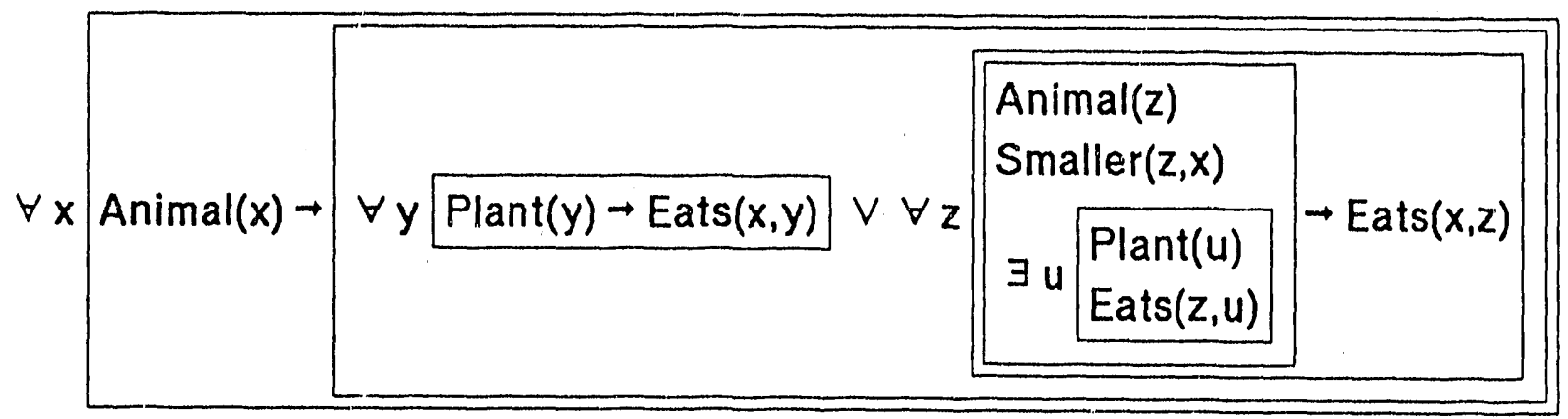

This memorandum describes a program that displays first-order logic formulas in the latter, more readable form.

The program also allows the user to perform two types of transformation on the displayed formulas. The logic transformations can be applied to the entire displayed formula or to a subformula and either produce an equivalent formula or preserve unsatisfiability. Examples of the logic transformations are conversion to conjunctive normal form, application of DeMcrgan's laws, and conversion to clauses. The edit transformations can be used to make arbitrary changes to the displayed formula. Examples are to negate a subformula, to delete a subformula, and to edit a subformula as a text string. FORMED keeps track of previous states, so that transformations can be "undone".

As of this writing, FORMED is not yet connected to a theorem prover, but we intend for it to become the interface to an interactive theorem prover and/or proof checker (which does not yet exist). However, it is useful now as a stand-alone program: 
1. to help students learn formal logic,

2. to study properties of normal-form conversion (the buttons CNF simp and DNF simp are decision procedures for validity and unsatisfiability, respectively, of propositional logic formulas), and

3. to help prepare input for a resolution/paramodulation theorem prover such as OTTER [2].

FormED was written using the $\mathrm{X}$ Window System, Version $11[4,1,3]$. X was chosen because it seems to be emerging as the standard graphics and windowing environment for high-performance workstations. The code for file input/output of formulas and for logic transformations on formulas was taken directly from the theorem prover OTTER [2].

This memorandum is primarily an overview and progress report, including some justification of design decisions. It can also serve as a users' guide, but FormeD's extensive on-line help facility should not be overlooked.

\section{Syntax}

When FORMED reads formulas from a file or writes formulas to a file, or when the user interactively edits formulas, the formula syntax of OTTER is used. The following rules are taken from the OTTER 2.0 Users Guide [2].

\subsection{Terms and Atoms}

Names are alphanumeric strings that may contain some other characters such as $\$$ and .. A name may contain $L_{i}$, to 50 characters. Names are used as constant symbols, function symbols, predicate symbols, propositional variables, and regular variables.

A name is a variable iff it is bound by a quantifier. Most complex terms are written in prefix form, for example, $f(a, b, c)$. Prolog-style list notation can be used to write terms that represent lists: the symbol [] is an abbreviation for $\$ n i],\left[t_{1} \mid t_{2}\right]$ an abbreviation for $\$$ cons $\left(t_{1}, t_{2}\right)$, and $\left[t_{1}, t_{2}, t_{3}, t_{4}\right]$ an abbreviation for $\$ \operatorname{cons}\left(t_{1}, \$ \operatorname{cons}\left(t_{2}\right.\right.$, $\left.\left.\$ \operatorname{cons}\left(t_{3}, \$ \operatorname{cons}\left(t_{4}, \$ n i l\right)\right)\right)\right)$. The notation $\left[t_{1}, t_{2} \mid t_{3}\right]$ is not allowed-such an expression must be written $\left[t_{1} \mid\left[t_{2} \mid t_{3}\right]\right]$.

White space (spaces, tabs, newlines) can occur in complex terms anywhere except within names and between a function or predicate symbol and the opening parenthesis.

Atoms are similar to complex terms, except that a name is also an atom (a propositional variable), and equalities and negated equalities can be written in infix form as 
$\left(t_{1}=t_{2}\right)$ and $\left(t_{1} \quad !=t_{2}\right)$. White space is required around $=$ and $!=$, and parentheses are required.

\subsection{Formulas}

1. Atoms are formulas.

2. If $F$ and $G$ are formulas, then $(F \leftrightarrow G)$ and $(F \rightarrow G)$ are formulas.

3. If $F_{1}, \ldots, F_{n}$ are formulas, then $\left(F_{1}|\ldots| F_{n}\right)$ and $\left(F_{1} \& \ldots \& F_{n}\right)$ are formulas.

4. The symbols all and exists are quantifiers. If $Q_{1} \ldots Q_{n}$ are quantifiers, $x_{1} \ldots x_{n}$ are names, and $F$ is a formula, then $\left(Q_{1} x_{1} \ldots Q_{n} x_{n} F\right)$ is a formula.

5. If $F$ is a nonnegated formula, then $-F$ is a formula.

The symbols have their expected meanings: - means "not", $\longleftrightarrow->$ means "if and only if", $\rightarrow$ means "implies", I means "or", and \& means "and".

All parentheses are required, and white space is required around $\langle->,->$,$| , and$ and after quantifiers and their associated variable occurrences.

Note that the following are not formulas: $-p(a)$ (double negation), ( $p \& q \rightarrow$ r) (not enough parentheses), (all $\times p(x) \& q(x))$ (not enough parentheses), and (p\&q) (not enough white space).

\section{Starting FormEd}

FORM ED recognizes three command line options:

-l filename Load formulas in the specified file during startup. Formulas can also br loaded after startup with the button Load in the main menu.

-f color Use the named color tor the foreground on color monitors (ignored on blackand-white monitors).

-b color Use the named color for the background on color monitors (ignored on blackand-white monitors). 
When Form ED starts, the following menu is displayed at the top of the new window.

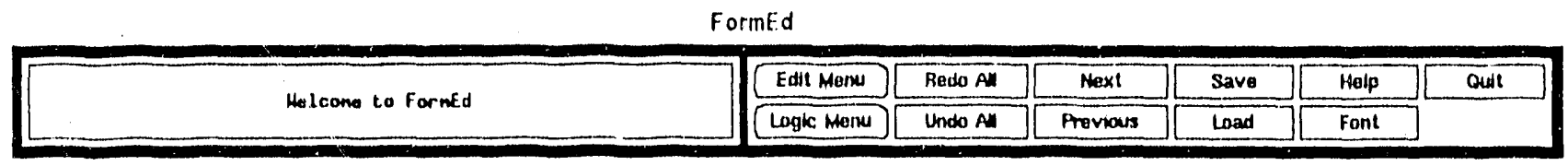

The actions of the buttons are as follows.

Quit Exit the program. FORMED does not check that the user has saved his or her work.

Help The on-line documentation is very useful.

Font The user can select from three font sizes for the display of formulas. The default is "medium".

Save Save the (transformed) formulas to a file.

Load Load a file of formulas. This causes the deletion of any formulas in FORMED at the time of the load.

Next, Previous Display the next or previous formula in the main formula list. If any transformations have been applied to the next or previous formula, the transformed version is displayed. (All steps leading to the transformed version are saved by the undo/redo mechanism. See Section 7).

Undo All Display the original formula. (See Section 7.)

Redo All Display the fully transformed formula. (See Section 7.)

Logic Menu Display the menu for logic transformations. (See Section 5.)

Edit Menu Display the menu for editing transformations. (See Section 6.)

\section{The Display and Selection of Subformulas}

Other than font size, the user has no control of the arrangement of formulas in the window. Conjunctions are displayed vertically, with no "\&" symbol separating the conjuncts. All other types of formula are displayed horizontally (with the appropriate logic symbol). To group subformulas, we use rectangles rather than parentheses or brackets. Our original intent was to use parentheses and/or brackets of varying sizes, but the consensus was that rectangles are easier to read. Conjunctions, disjunctions, implications, and equivalences are enclosed in rectangles; atoms, negations, and quantified formulas are not. 
Most of the logic and editing operations can be applied to subformulas of the displayed formula as well as the entire formula. The Operate logic transformation applies to logic symbols $[\neg, \rightarrow, \leftrightarrow]$ rather than formulas. The user selects a subformula or a logic symbol by simply clicking on the subformula or symbol. The selected area (always a rectangle) of the display is highlighted in reverse video. To "unselect" an area without selecting another, one simply clicks either on the selected area or outside of the entire formula. If no area of the disptay is highlighted, the entire formula is selected by default. An atomic formula (a propositional variable or a predicate symbol applied to a sequence of terms) is atomic with respect to selection.

If the displayed formula does not fit in the window, a smaller font can be selected by pressing the button Font. If scroll bars are present, they can be used in the normal way.

Technical Detail. The canvas is the area onto which the formula is drawn, and the viewport contains the part of the canvas that is displayed on the screen. If the canvas is larger than the viewport, scroll bar's should be present and should allow viewing of all parts of the canvas. If the formula being drawn does not fit on the canvas, it is simply truncated. The size of the canvas is fixed at compile time. To enlarge (or shrink) the canvas, the user can change the definitions of CANVAS_HEIGHT and/or CANVAS_WID'TH in the source file formed.h. (A large canvas can use a lot of memory, especially on a color monitor. For example, a $4000 \times 4000$ canvas on a Sun color monitor requires 16 megabytes.)

\section{$5 \quad$ Logic Transformations}

When the user presses the button Logic Menu in the main menu, the logic menu is displayed to the left of the main menu:

FormEd

\begin{tabular}{|c|c|c|c|c|c|c|c|c|c|c|}
\hline Causify & NNF & CNF & DNF & Redo & Edit Merw & Rodo AM & Next & Save & Help & Quit \\
\hline Operate & Skolemize. & CNF skmp & DNF $\sin p$ & Undo & Logic Menu & Undo All & Provious & Load & Font & \\
\hline
\end{tabular}

Recall that if no area of the displayed formula has been selectud (highlighted), the entire formula is considered to be selected. All of the logic transformations except Clausify and Skolemize produce a logically equivalent formula. The functions of the buttons are as follows.

Clausify The entire formula must have been selected. The formula is transformed into a conjunction of clauses by the following steps. Convert to negation normal form (NNF), Skolemize, make all universally quantified variables unique, delete quantifiers, rename variables so that they start with " $u$ "-" $z$ ", then con- 
vert to conjunctive normal form (CNF). Nothing should be done with the resulting clauses, because at the data structure level, they are in limbo between the formula world and clause world. Further logic transformations may be unsound, because quantifiers have been deleted, and OTTER will not accept them as clauses (OT'TER can clausify the formula instead). The main purpose of Clausify is just to see the "clauses". Clausification preserves unsatisfiability.

Skolemize The entire formula must have been selected. (It is sometimes useful to Skolemize part of a formula, but ForMED does not have that capability.) The formula is first converted to negation normal form (NNF); then it is Skolemized. Skolemization preserves unsatisfiability.

Operate One of the logic symbols $[\neg, \rightarrow, \leftrightarrow]$ must have been selected. This button causes a one-level (nonrecursive) operation to be applied.

$\neg$ If the negation sign applies to a conjunction, disjunction, or quantified formula, the negation sign is moved inward one level.

$\rightarrow$ The implication $(F \rightarrow G)$ is converted to $(\neg F \vee G)$.

$\leftrightarrow$ The formula $(F \leftrightarrow G)$ is converted to either a conjunction $\left[\begin{array}{l}F \rightarrow G \\ G \rightarrow F\end{array}\right]$ or a disjunction $\left[\begin{array}{l}F \\ G\end{array}\right] \vee\left[\begin{array}{l}\neg F \\ \neg G\end{array}\right]$. The user is prompted for his or her choice.

NNF The selected formula is converted to negation normal form by moving negation signs inward and removing $\rightarrow$ and $\leftrightarrow$. In the resulting NNF formula, all negation signs are against atomic formulas. When removing equivalences and negated equivalences, conjunctions are always preferred, because the goal is usually conjunctive normal form.

CNF The selected formula is converted to a conjunctive normal form (CNF, a conjunction of disjunctions). Subformulas of any quantified formulas are also converted. No simplification is attempted during conversion.

CNF simp The selected formula is converted to simplified conjunctive normal form. Less general (including tautological) disjunctions are removed. Subformulas of any quantified formulas are also converted. This button is a decision procedure for validity of propositional formulas.

DNF The selected formula is converted to a disjunctive normal form (DNF, a disjunction of conjunctions). Subformulas of any quantified formulas are also converted. No simplification is attempted during conversion.

DNF simp The selected formula is converted to simplified disjunctive normal form. More general (including contradictory) conjunctions are removed. Subformulas of any quantified formulas are also converted. This button is a decision procedure for unsatisfiability of propositional formulas. 
Undo, Redo All steps from the original formula to the current formula are saved so that the transformations can be "undone" if necessary. The "undone" transformations are also saved so that they can be "redone" if necessary. These buttons apply only to logic transformations. (If logic and editing transforma. tions are mixed, one may have to go to the logic menu and "Undo", then go to the edit menu and "Unedit", etc. See Section 7.)

\section{Editing Transformations}

When user the presses Edit Menu in the main menu, the edit menu is displayed to the left of the main menu:

Formed

\begin{tabular}{|c|c|c|c|c|c|c|c|c|c|c|}
\hline Edill & Conjoln & cxuandfy & New formuda & Ro-edift & Edil Mernus & Redo Ax & Mext & Save & Help & Qunt \\
\hline Abbrevilate & Dtsjoin & Megale & Deteto formula & Unedil & Logle Menul & Undo All & Provitous & Load & Font & \\
\hline
\end{tabular}

Several of the editing operations require the user to type in formulas or to change a text string. When that occurs, a separate window containing a text area pops up. Some basic Emacs commands are used to edit and navigate through the text area. For FormED vi,ers who do not use Emacs, the essential Emacs operations are

control-f

control-b

your erase character

control-d

Tyning characters inserts them before the cursor.

Move the cursor forward one character.

Move the cursor backward one character.

Delete the character behind the cursor.

Delete the character ahead of the cursor.

The operation Edit is the most general editing operation, and we present here an example of its use. The following picture shows FORMED in the middle of an 
Edit operation.

FormEd

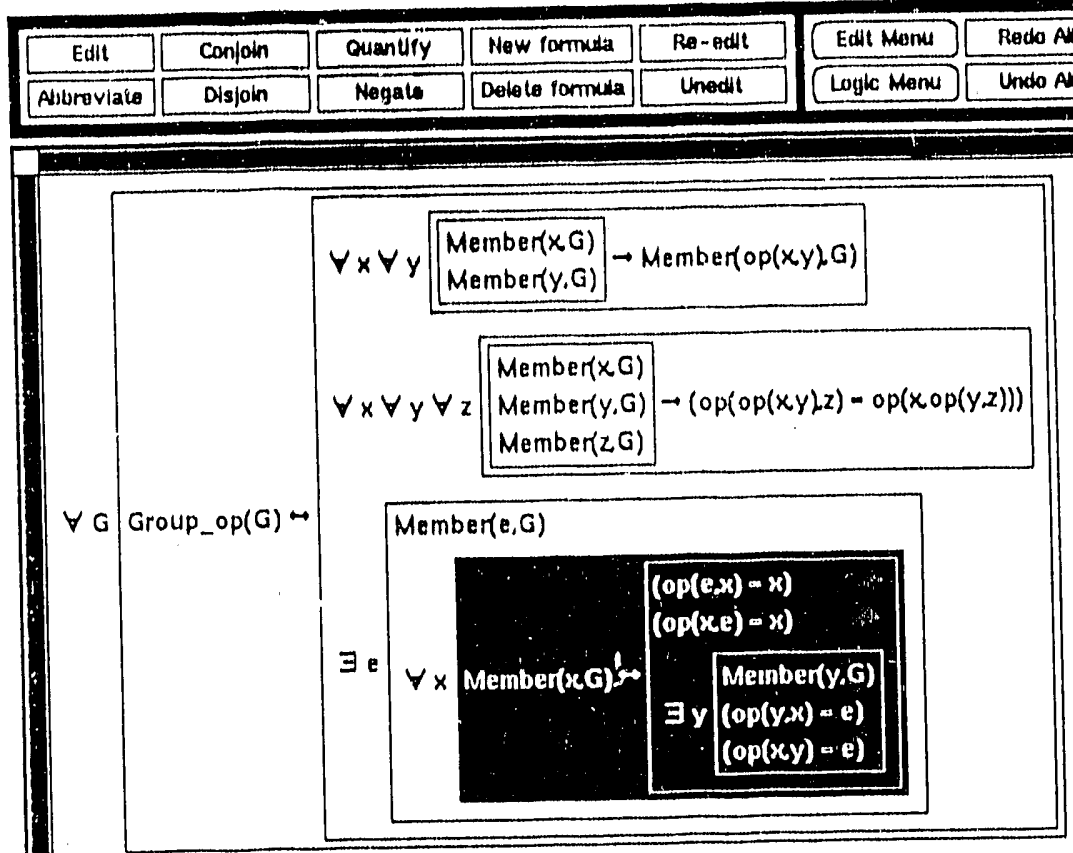

\begin{tabular}{|c|c|}
\hline Replace & \multirow{3}{*}{ 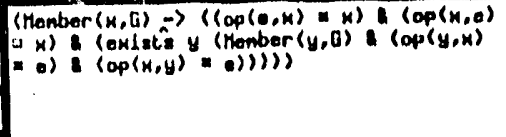 } \\
\hline dear & \\
\hline Cancel & \\
\hline & \\
\hline & \\
\hline
\end{tabular}

We wished to change $\leftrightarrow$ to $\rightarrow$ in the highlighted area, so we selected that area and pressed Edit. The window ccntaining the text string (OTTER) form of the highlighted area popped up; then we made the change in the text string. Pressing Replace in the popup window will then cause the transformation to take effect. If any syntax errors are detected, a message is sent to the standard output (the window from which FORMED was invoked); then the user can correct the mistake. (Error handling should be improved in future versions of FORMED.)

The editing operations are as follows.

Edit The user edits the selected formula as a text string. See the preceding paragraph.

Abbreviate This button is not yet operational. Eventually it will allow the user to abbreviate a subformula of the displayed formula. 
Conjoin An empty text area pops up, and the user types in a forrnula. The formula is then conjoined with (and'ed on to) the selected formula.

Disjoin An empty text area pops up, and the user types in a formula. The formula is then disjoined with (or'ed on to) the selected formula.

Quantify An empty text area pops up, and the user types in quantifiers and variables, for example, "all $x$ exists $y$ all $z$ ", which are prepended to the selected formula.

Negate The selected formula is negated.

New Formula An empty text area pops up, and the user creates a new formula. The new formula is inserted after the current formula.

Delete Formula If the entire displayed formula is selected, it is deleted. Otherwise, an immediate subformula of a conjunction or a disjunction must be selected; the subformula is deleted from the conjunction or disjunction.

Unedit, Re-edir All steps from the original formula to the current formula are saved so that the transformations can be "undone" if necessary. The "undone" transformations are also saved so that they can be "redone" if necessary. These buttons apply only to editing transformations. (If logic and editing transformations are mixed, one may have to go to the logic menu and "Undo", then go to the edit menu and "Unedit", etc. See Section 7.)

\section{The Undo/Redo Mechanism}

Six buttons can be used to control backtracking and retracing of steps through transformations:

Undo All, Redo All in the main menu.

Undo, Redo in the logic menu.

Unedit, Re-edit in the edit menu.

A doubly linked list of formulas is maintained. The list starts with the original formula and ends with the final transformation. The disp'ayed formula can be any member of the list. The buttons Undo A.11, Redo All in the main menu display the first and last, respectively, members of the list.

Suppose the user makes four transformations (of either type, including mixed) on an original formula, then "undoes" two of the transformations (with the appropriate buttons). Then the third member of the list is displayed. If the user then makes a transformation, the fourth and fifth members of the list are deleted (otherwise a tree 
would be required), and new fourth member (the transformed formula) is appended and displayed.

Since logic and editing transiornations can be mixed, and it is probably wise to keep them concepiually apart, the list of formulas is two dimensional: logic transformations are horizontal, and editing operations are vertical.

Suppose the user has made the following types of transformation on an original formula: 2 logic, 1 edit, 1 logic, 2 edit, 1 logic. Then the state of the list is as follows.

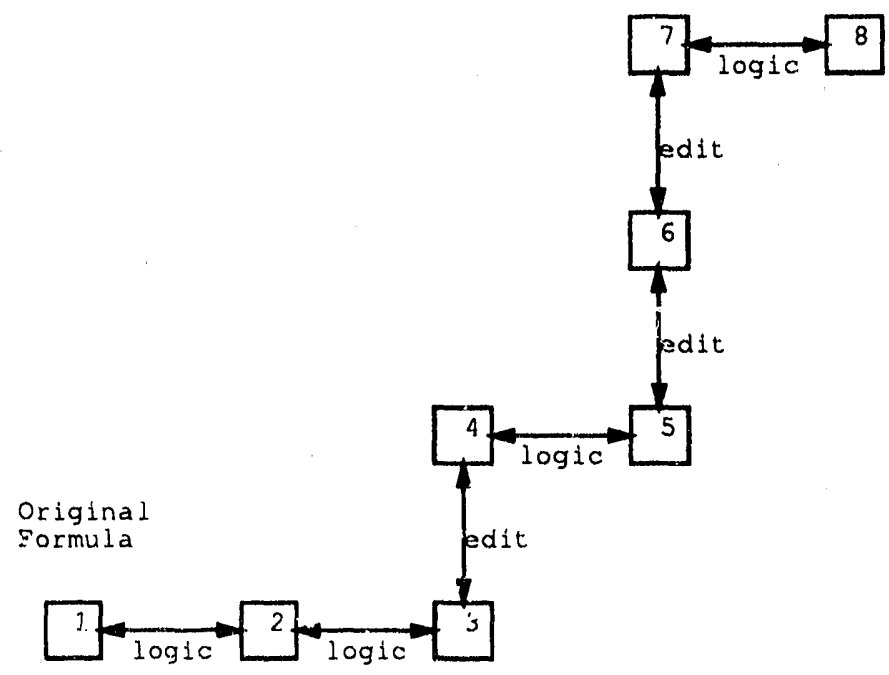

The buttons Undo, Redo in the logic menu rove left and right, respectively, in the list. The buttons [Unedit, Re-edit] in the edit menu move down and up, respectively, in the list.

\section{The Display and Select Algorithms}

Displaying a formula on the canvas consists of two recursive procedures: Arrange and Draw. A formula is a tree with atoms as the leaves and logic symbols as internal nodes. (Nodes that represent quantified formulas are a bit different, but we ignore that fact to simplify the presentation.)

The three types of object we draw are text strings (atoms and quantified variables), logic: symbols, and rectangles. We know the sizes (in pixels) of the logic symbols; X routines can tell us the size (in pixels) of text strings.

The procedure Arrange lays out the formula (it does not draw anything on the canvas) by traversing the formula bottom "up and building a layout tree similar to the formula. The layout tree differs from the formula by having leaves for the logic symbols. Each node of the layout tree represents a rectangular space. Leaves are text strings and logic symbols, and internal nodes are composite rectangles. Each node of the complete layout tree contains the size of the corresponding rectangle and the horizontal and vertical offsets from its parent's rectangle. To build a layout tree 
for a formula, one first builds the layout trees for the subformulas and logic symbols. Then the sizes of the child rectangles are considered, and their positions relative to one another and to their new parent-vertically, left-justified for conjunctions, and horizontally, centered for all others-are calculated and assigned to them. A new parent layout node is then allocated and connected to its children, and the size of the new rectangle is assigned to the new layout node.

The procedure Draw takes a layout tree and draws it top-down, starting at a griven position on the canvas. A parent knows the offsets for its children, so it can tell them where to draw the mselves. The actual rectangles are drawn for conjunctions, disjunctions, implications, and equivalences, but not for atoms, negations, or quantified formulas.

When the user clicks on a point in the canvas, the smallest rectangle corresponding to a layout node and containing the point is "selected" and highlighted. The procedure Select does that by traveling directly from the root of the layout tree to the appropriate node rather than traversing the entire layout tree. At a given node in the layout tree, the procedure Select asks its children whether they contain the point. If no child contains the point, the given layout node is returned; if a child contains the point (at most one child can contain it), a recursive call is made on that child.

\section{Extension to Terms}

Atomic formulas are currently displayed as simple text strings. An obvious and useful extension to FORMED would be to extend the layout to the term level, including set theory symbols, summations, and arrays. One goal would be for FORMED to have the capability of $T_{E X}$ math mode. (We investigated to some extent the direct use of $\mathrm{T}_{\mathrm{E} X}$, but we were unable to find the appropriate primitives to display PosiScript or .dvi files on the canvas. It also seemed likely that intermediate file handling would be required during display, and that would be too slow for us: )

The current method of adding a new symbol to FORMED's repertoire is cumbersome. We must create the symbol in three different sizes with the bitmap editor, then make many additions to the $\mathrm{C}$ code to initialize, layout, etc. A more convenient method is required.

\section{References}

[1] J. McCormack, P. Asente, and R. R. Swick. X Toolkit Intrinsics - C Language Interface, $X$ Window System, $X$ Version 11, Release 4. MIT Press, Cambridge, MA, 1985. 
[2] W. McCune. OTTER 2.0 users guide. Tech. Report ANL-90/9, Argonne National Laboratory, Argonne, IL, March 1990.

[3] C. D. Peterson. Athena Wiget Set - $C$ Language Interface, $X$ Window System, $X$ Version 11, Release 4. MIT Press, Cambridge, MA, 1985.

[4] D. A. Young. $X$ Window Systems: Programming and Applications with Xt. Prentice-Hall, Englewood Cliffs, NJ, 1989. 

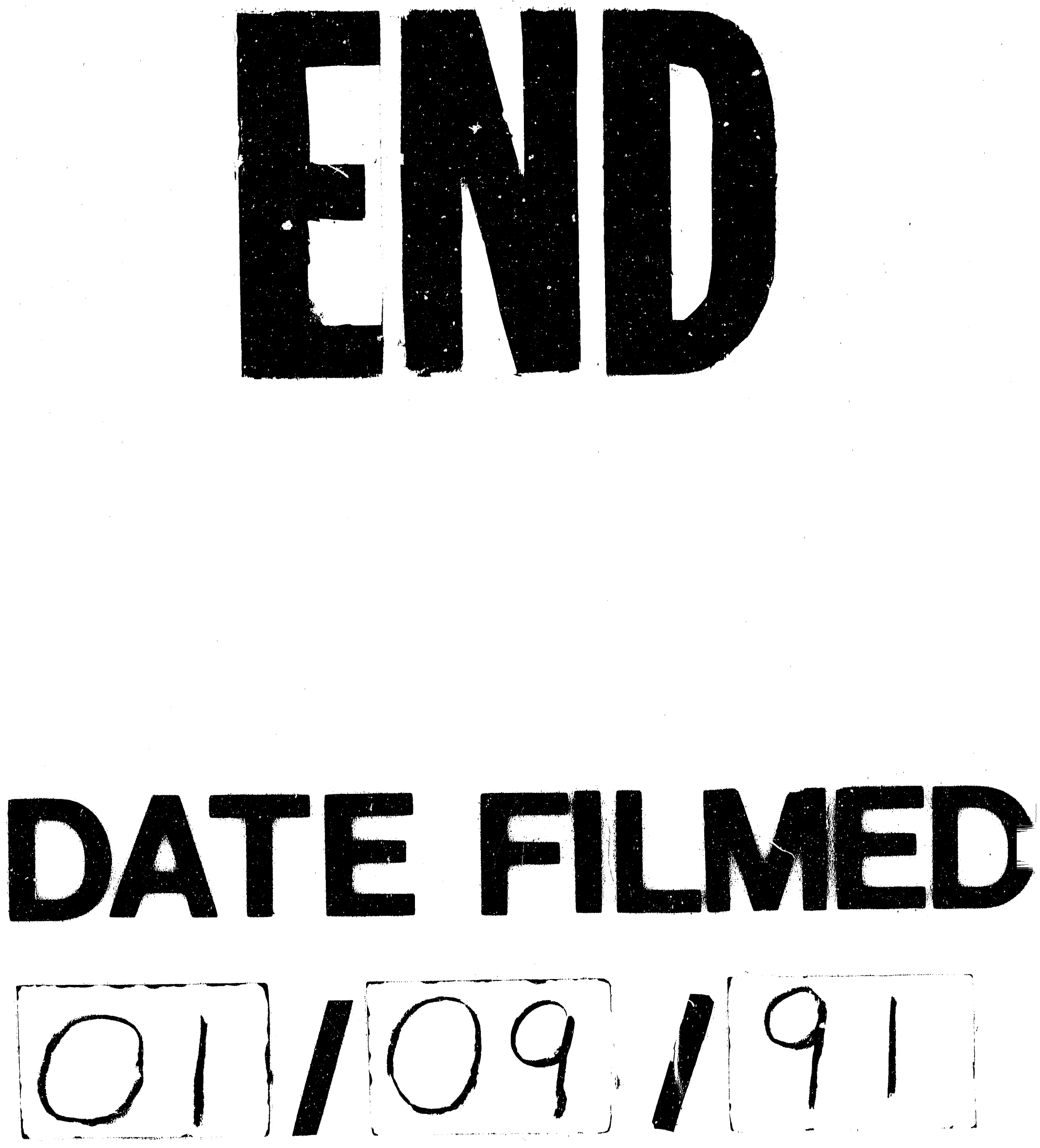
$=$ 